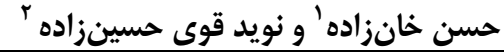

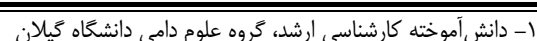

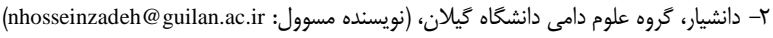

تاريخ دريافت: •

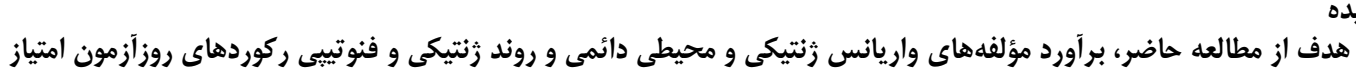

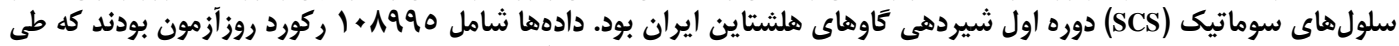

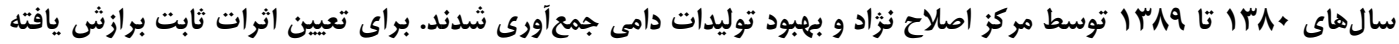

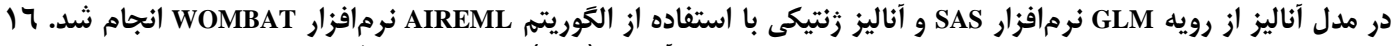

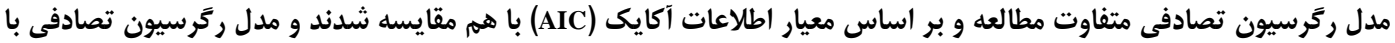

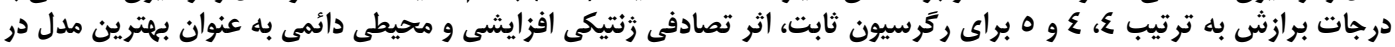

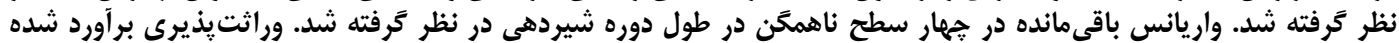

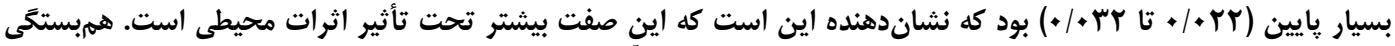

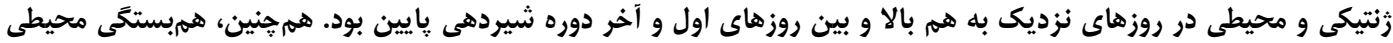

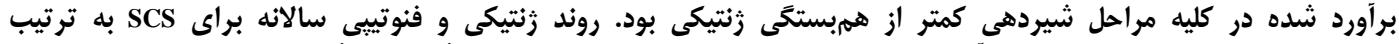

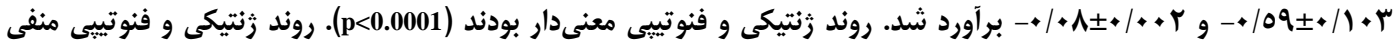

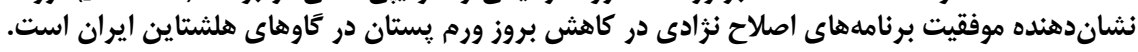

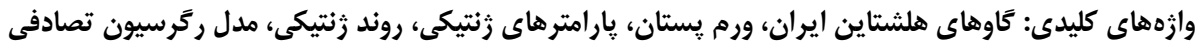

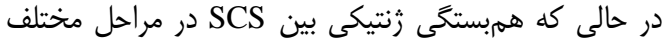

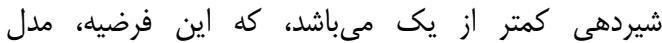

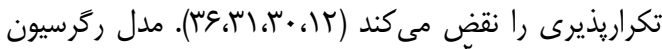

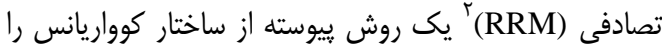

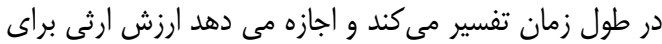

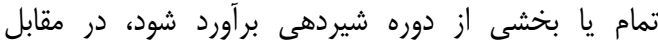

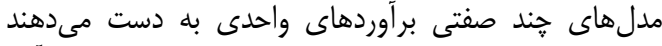

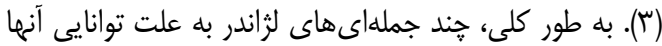

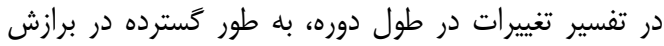

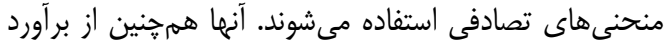

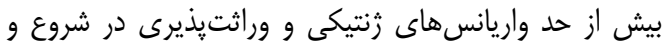

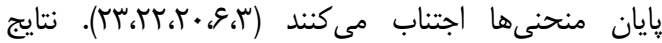

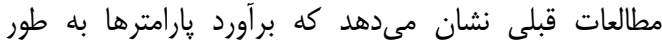

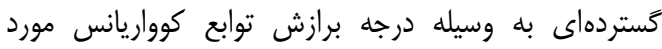

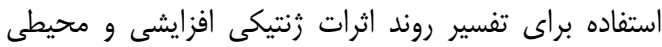

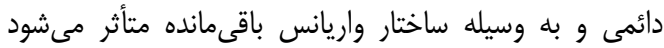

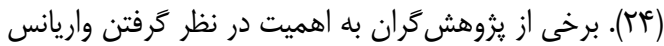

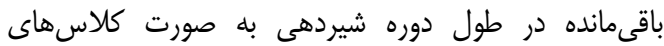

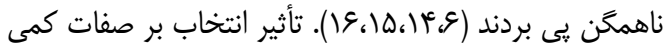

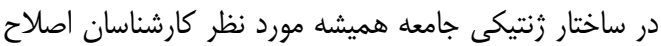

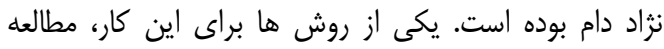

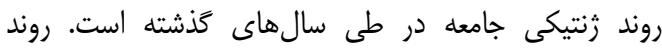

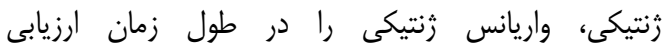

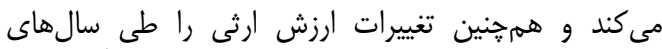

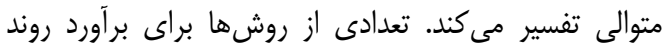
رنتيكى در جمعيت كاوهاى شيرى در دسترس است است، مانند

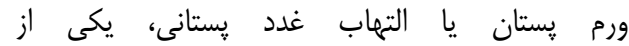

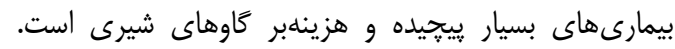

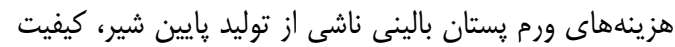

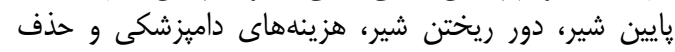

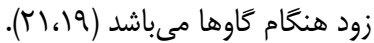

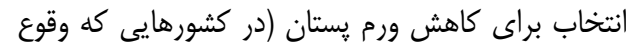

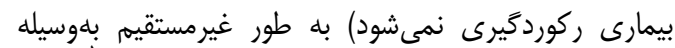

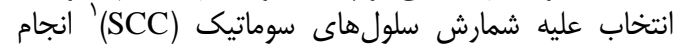

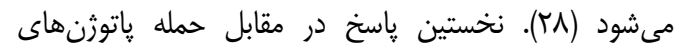

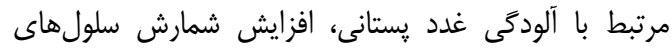

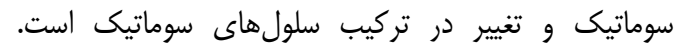

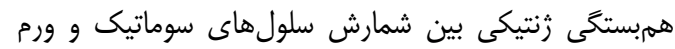

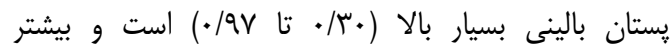

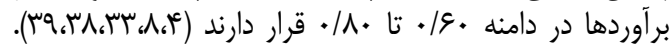

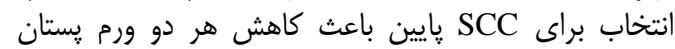

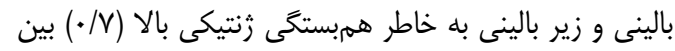

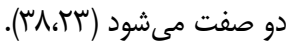
بيشتر كشورها هر دو صفت توليد شئ شير و مقار مقاومت در مقابل

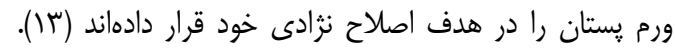

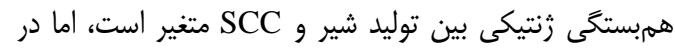

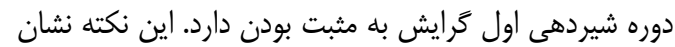

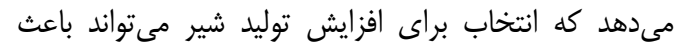

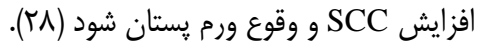

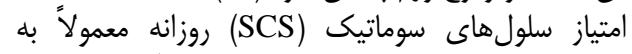
عنوان اندازمَيرىهاى تكرار شده از يك صفت آناليز مى شود، 
ايالات متحده و كانادا تأمين مىشوند (I) (I). ارزش SCS براى دامها بر اساس رابطه (I) محاسبه شد (IVI) (IV): $\mathrm{SCS}=\log _{2}(\mathrm{SCC} / 100)+3$ رابطه (1)

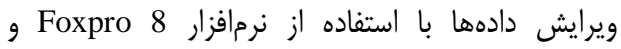

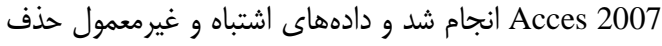

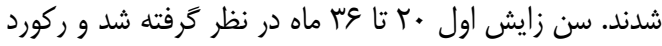

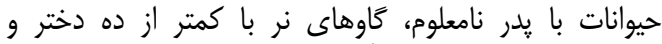

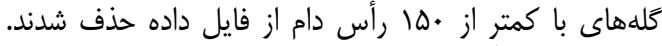

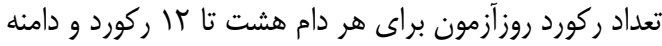

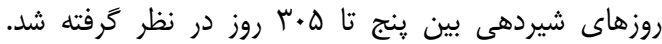

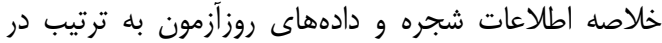

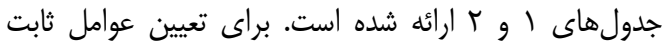

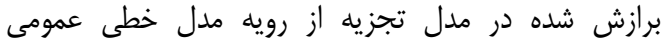

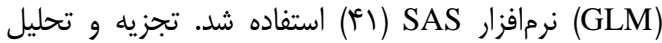

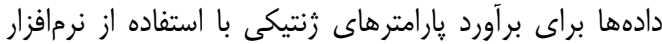

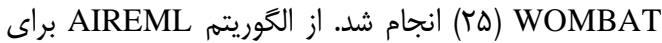
برآورد اجزاى واريانس استفاده شد.
آزمايشهاى انتخاب برنامهريزى شده يا استفاده از دادههاى

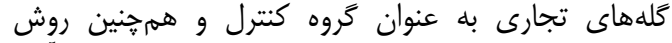

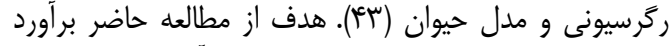

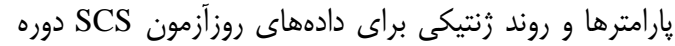

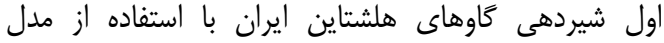
ركر سيون تصادفى است.

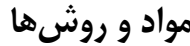

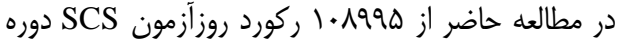

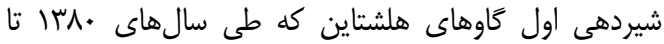

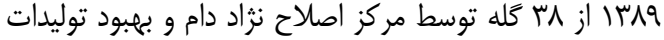

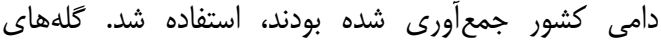

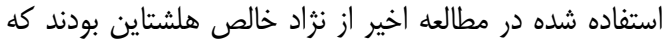

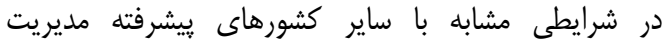

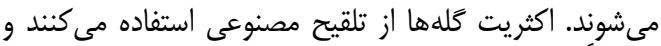

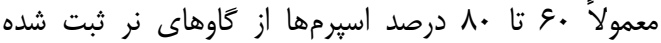

Table 1. Summary of pedigree information

جدول ا- خلاصهاى از اطلاعات شجره

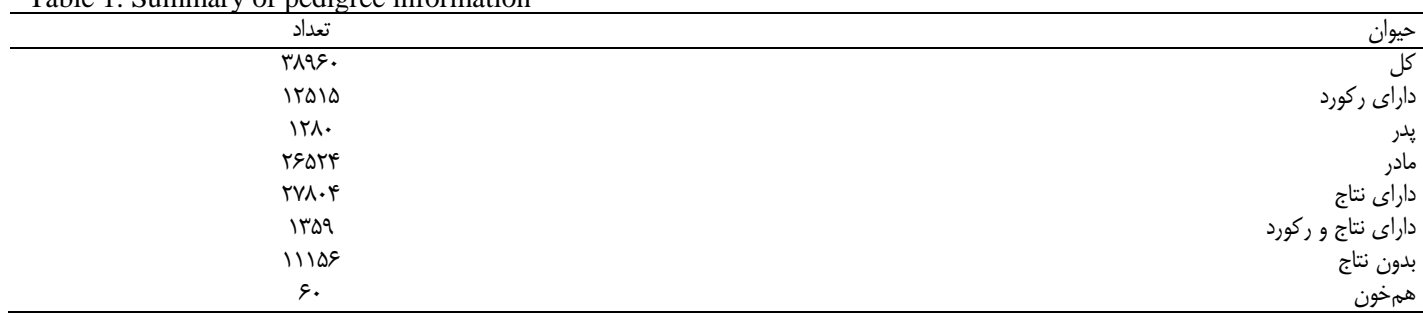

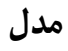

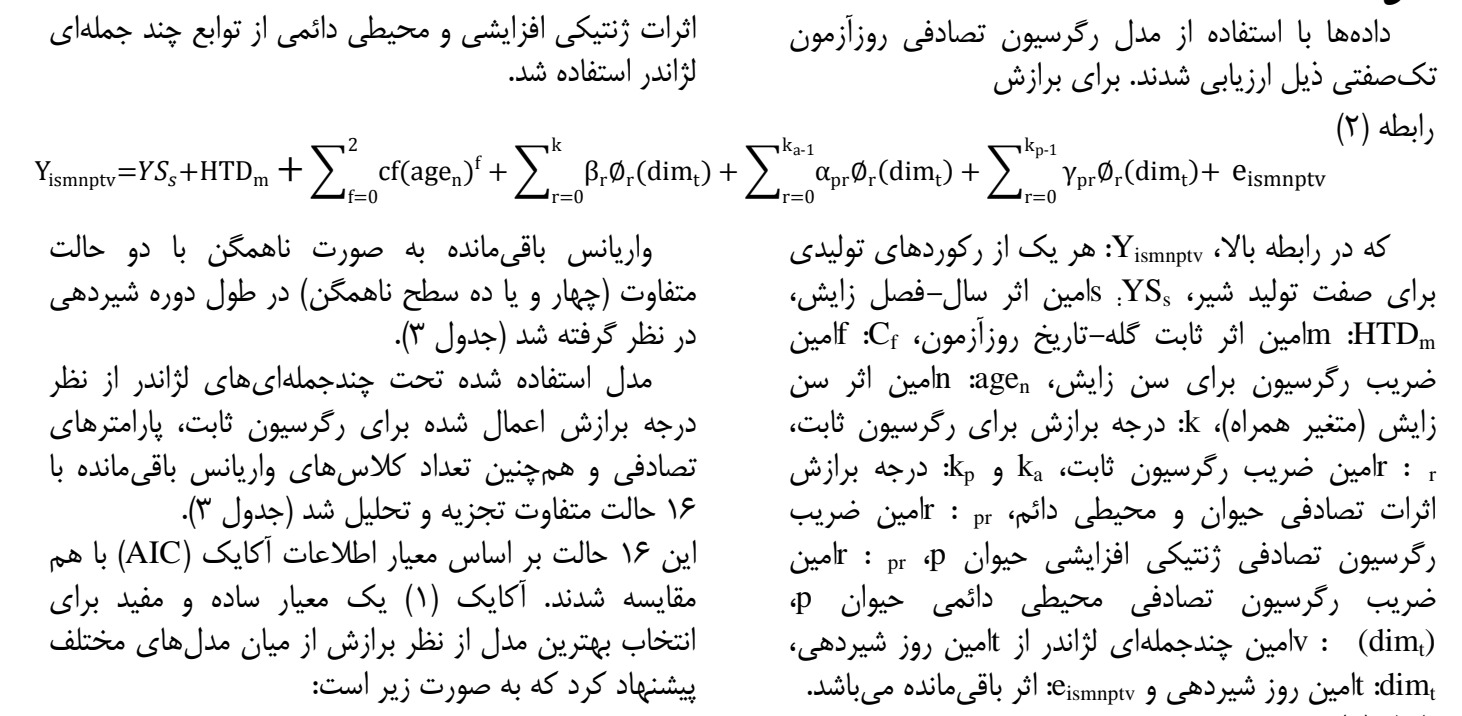

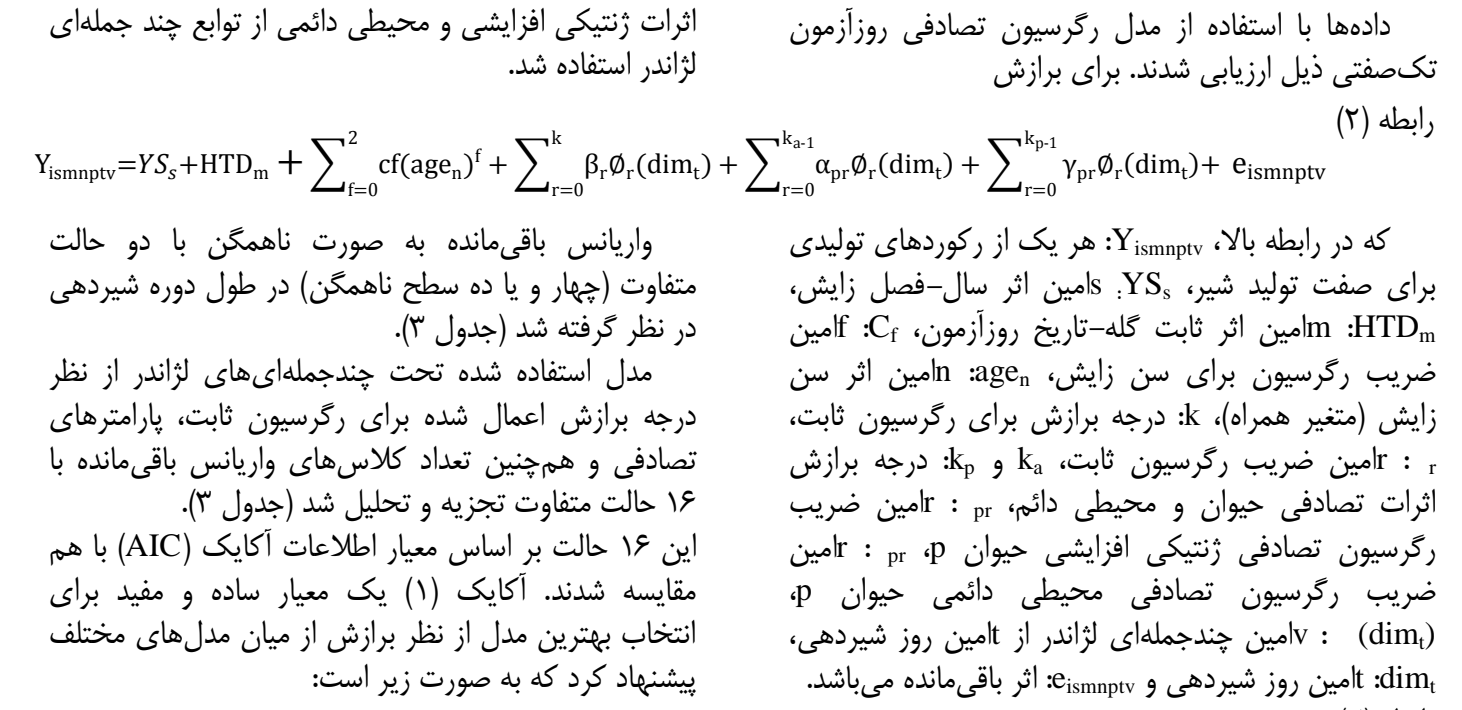

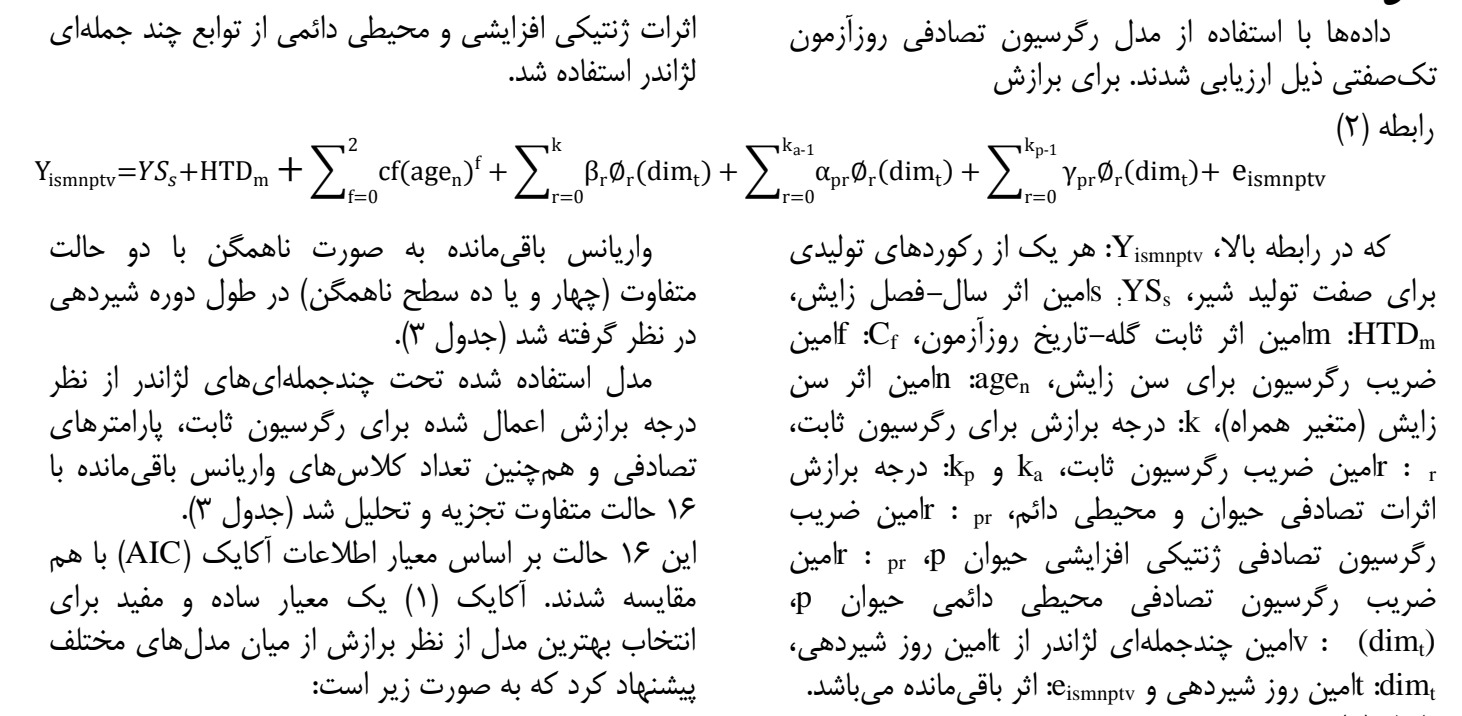

AIC $=-2 \log ($ maximum likelihood) +2 (number of model paramers)

انتخاب مىشود. آزمايشات متفاوتى كارايى AIC درايى در انتخاب

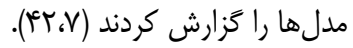

تفاوت بين AIC مدلهاى مختلف مهرم است، نه اندازه

محض AIC ها. مدل با كمترين AIC به عنوان بهترين مدل ملن الناره 
Table 2. Summary of test-day records information

\begin{tabular}{|c|c|c|c|c|c|c|}
\hline \multicolumn{2}{|c|}{ SCS } & \multicolumn{2}{|c|}{ SCC } & \multirow{2}{*}{ تعداد ركورد } & \multirow{2}{*}{ روزهاى شيردهى } & \multirow{2}{*}{ روزآزمون } \\
\hline انحراف معيار & ميانكين & انحر اف معيار & ميانكين & & & \\
\hline$T / N T$ & $r / r q$ & $\Gamma \Delta T / \cdot \Delta$ & $10 \Delta / R^{\prime} q$ & $1 \cdots \Delta 9$ & $r \Delta-\Delta$ & 1 \\
\hline $1 / \mathrm{r}$ & $1 / 9$. & rev/qT & | |r/ & $11 f+1$ & 9Q_K & r \\
\hline $1 / \mathrm{Vr}$ & $1 / 19$ & MFN/T. & 落/هD & אזrוI & $90-99$ & r \\
\hline $1 / V V$ & 1/98 & $r \Delta q / 1$. & $1 f \cdot / 4 \Delta$ & $11 V \cdot \Delta$ & $\mid r \Delta-q \varepsilon$ & f \\
\hline $1 / v 9$ & $r / .$. & TET/.q & $148 / 99$ & זגזוI & $1 \Delta D-I \Gamma \varphi$ & $\Delta$ \\
\hline $1 / \mathrm{r}$ & $r / .9$ & $r \Delta \cdot / \Delta \Lambda$ & سו//Fם & $\| V \Delta \Delta$ & $\mid \Lambda \Delta-1 Q \Phi$ & 9 \\
\hline $1 / \wedge$. & זו/ץ & $r \Delta F / l$. & IQT/VQ & גזrוI & $r \mid Q-\backslash N E$ & v \\
\hline $1 / 11$ & T/R & $r V \Delta /{ }^{\prime} q$ & $191 / N$ & זוצו| & $r \Psi \Delta-r \mid \varphi$ & $\wedge$ \\
\hline $1 / V V$ & $r / \mu$ & 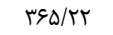 & $19 T / T V$ & $1.9 \cdot 0$ & TVQ_TES & 9 \\
\hline $1 / v 9$ & $r / s \Delta$ & ו & $|Y N / r|$ & VAls & $r \cdot \Delta-r V G$ & 1. \\
\hline $1 / v \wedge$ & $r / I$ & rVQ/\&V & $199 / 19$ & $1.19 \Delta \Delta \Delta$ & $\Gamma \cdot \Delta-\Delta$ & كل \\
\hline
\end{tabular}

Table 3. Heterogeneous classes for residual variance

\begin{tabular}{|c|c|c|c|c|}
\hline \multicolumn{2}{|c|}{ حالت دوم } & \multicolumn{2}{|c|}{ حالت اول } & \multirow{2}{*}{ سطح } \\
\hline روز پايان & روز آغاز & روز هايان & روز آغاز & \\
\hline$r$. & $D$ & $r$. & $\Delta$ & 1 \\
\hline r. & ו" & Ir. & ו" & $r$ \\
\hline 9. & 91 & Tr. & $|\pi|$ & r \\
\hline IT. & 91 & $r \cdot \Delta$ & THI & f \\
\hline 10. & $|K|$ & & & Q \\
\hline $1 \Lambda$. & $|0|$ & & & 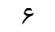 \\
\hline r). & $|1|$ & & & $\checkmark$ \\
\hline rF. & rII & & & 1 \\
\hline$r V$. & TEI & & & 9 \\
\hline$\mu \cdot \Delta$ & rn & & & 1. \\
\hline
\end{tabular}

جدول سـ- كلاسهاى ناهمخن براى واريانس باقىمانده

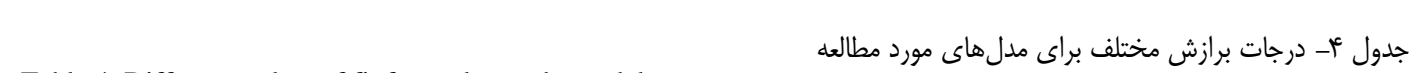

Table 4. Different orders of fit for under study models

\begin{tabular}{|c|c|c|c|c|c|c|c|}
\hline AIC & $\log _{1}$ & تعداد پِارامتر & واريانس باقيى بانداى & $\mathrm{kp}$ & $\mathrm{ka}$ & ردر دريون برازش ثابت & مدل \\
\hline T. HYT/KA & $V \leftarrow / 1.1990-$ & 18 & f & $r$ & $r$ & $\mu$ & 1 \\
\hline 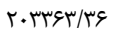 & SN1.19QQ- & r & 1. & $r$ & r & r & r \\
\hline$r \cdot r r \cdot \Delta / \Delta$. & $V Q / 1 \cdot \mid \Delta A Y-$ & $r$. & f & f & r & r & r \\
\hline$T \cdot r \cdot T / M \Lambda$ & $\cdot 9 / 1 \cdot 10 V \Delta-$ & ז & 1. & r & r & r & f \\
\hline$T \cdot r T \cdot / V T$ & 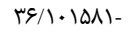 & re & f & f & f & r & $\Delta$ \\
\hline$T \cdot r \cdot T \cdot V / T \Lambda$ & $94 / 1 \cdot 1 \Delta V{ }^{\prime}-$ & $r$. & 1. & f & t & r & 9 \\
\hline$T \cdot M I T=/ A V$ & $|F(\mid) \cdot| \Delta F \mid-$ & rq & f & $\Delta$ & r & r & v \\
\hline$r \cdot r / \Delta q / I r$ & $\Delta S V / 1 \cdot \mid D F Y-$ & ra & 1. & $\Delta$ & i & r & $\wedge$ \\
\hline 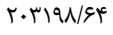 & TT/I.।QAT- & 19 & f & $r$ & r & f & 9 \\
\hline T.MI৭9/D. & $V Q / 1 \cdot 1 \Delta V V-$ & r & 1. & $r$ & r & f & 1. \\
\hline$r \cdot r \cdot F T / V G$ & $M N 1 \cdot 10 \cdot 1-$ & r. & f & r & r & f & 11 \\
\hline$T \cdot r \cdot F T / V T$ & $r q / .1 F q \Delta-$ & rq & 1. & f & r & f & Ir \\
\hline$r \cdot r \cdot r q / g r$ & $11 / 1 \cdot 10 \cdots$ & re & f & f & r & f & r \\
\hline$r \cdot r \cdot \Gamma / N / F \Lambda$ & $r F / 1 . \mid f q F_{-}$ & r. & 1. & r & i & f & if \\
\hline$r \cdot r q q . / r \pi$ & $114 \pi \cdot 1499$ & rq & f & 0 & i & f & 10 \\
\hline$r \cdot r q q Y / \cdot \Lambda$ & . FT/I. IEST- & ra & 1. & 0 & t & f & 19 \\
\hline
\end{tabular}

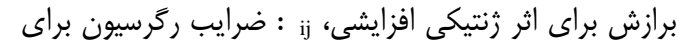

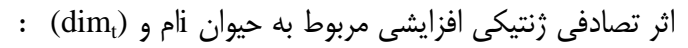

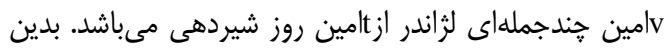

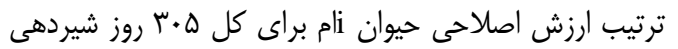

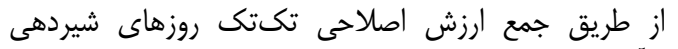

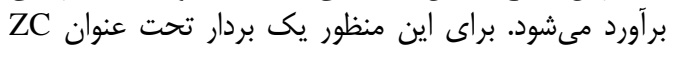

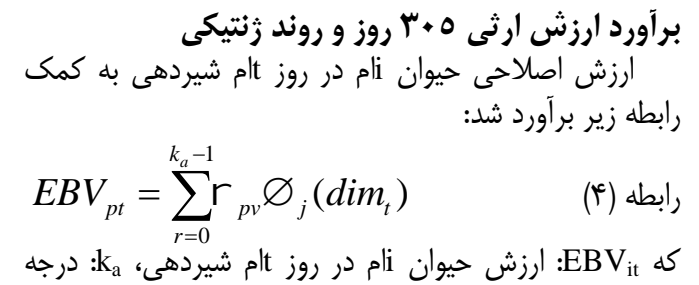


زرنتيكى و محيطى دائمى باشد، درجات برازش متفاوت براى تراى

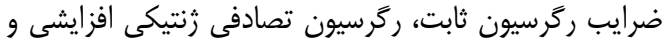

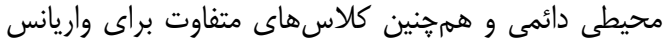

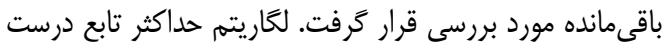

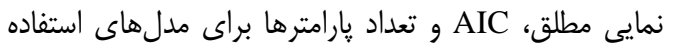

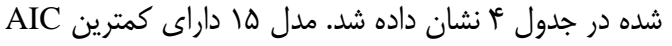

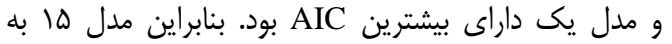
عنوان بهترين مدل انتخاب شداند. وراثتيذيرى و اجزاى واريانس - كوواريانس

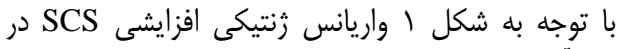

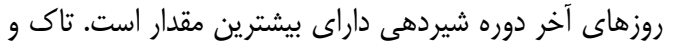

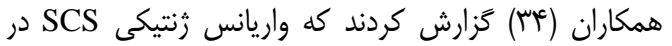

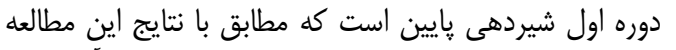

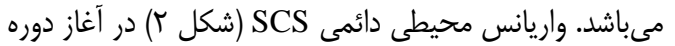

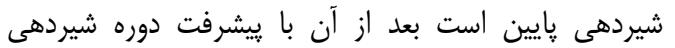

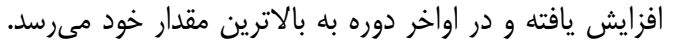

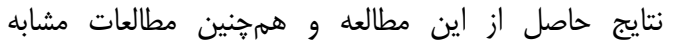

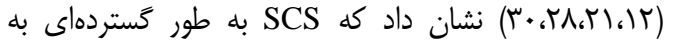
وسيله عوامل محيطى موقت متأثر مىشود و يكى معيار ثابت نابت براى يك كاو نيست.

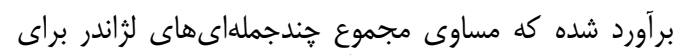
هر درجه برازش است. ZC برآورد شده در اين به صورت زير لرانير

بود:

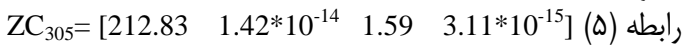

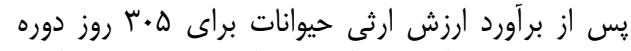

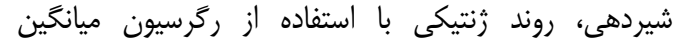

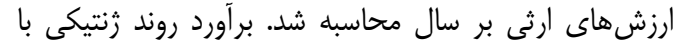

استفاده از رويه ركر سيون نرمافزار SAS انجام شد (أثان).

\section{نتايج و بحث}

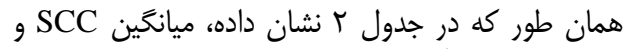

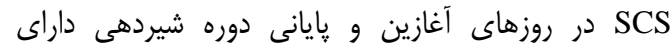

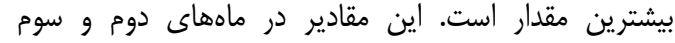

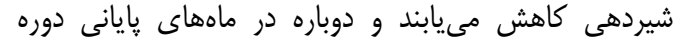

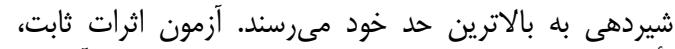

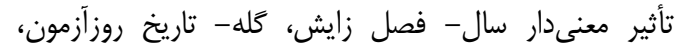

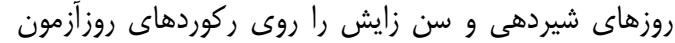

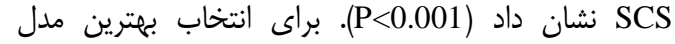

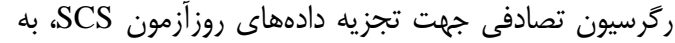

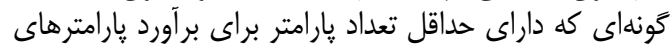

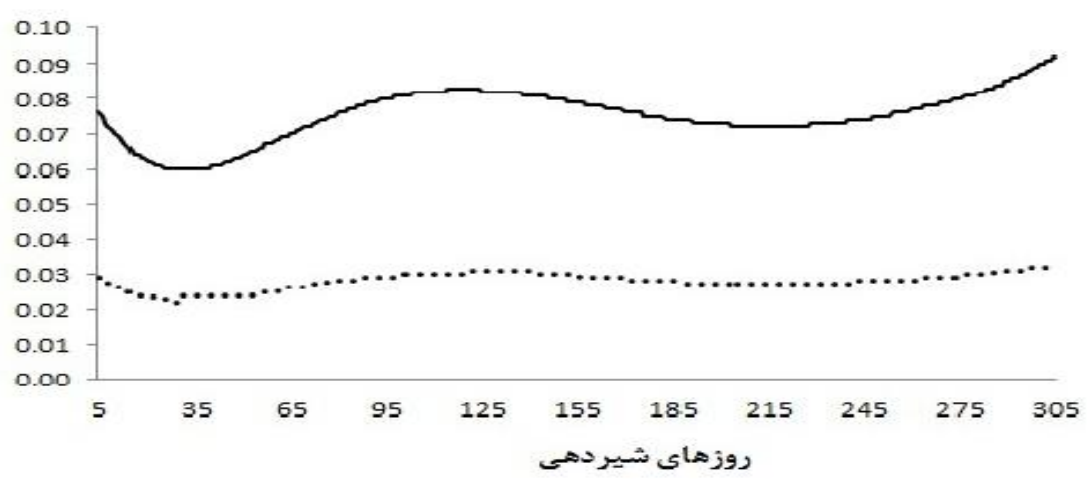

شكل ا- واريانس زنتيكى افزايشى (خط ييوسته) و وراثتيذيرى (نقطه هֶين) برآورد شده براى ركوردهاى روزآزمون SCS در طول دوره Figure 1. Additive genetic variance (continuous line) and estimated heritability (dot) for test-day records of SCS over
the lactation period

كردند كه تغييرات SCC در شروع دوره شيردهى احتمالاً به

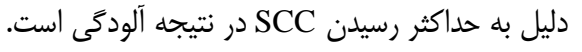

واريانس باقىمانده (جدول ه) در روزهاى آغازين دوره

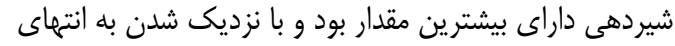

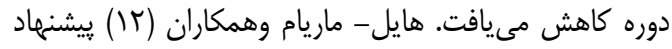

جدول ه- بر آورد واريانس باقى مانده براى كلاسهاى مختلف روزهاى شيردهى Table 5. Estimated residual variance for different classes of days in milk

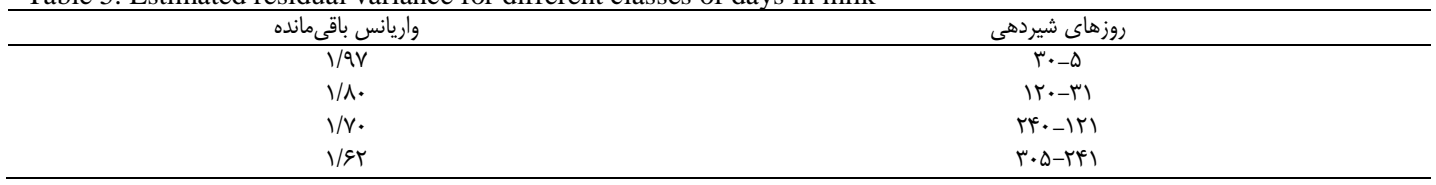




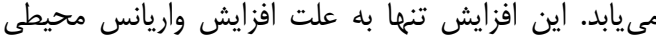

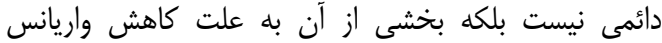

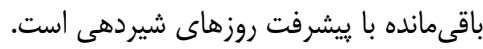
همبستخى زنتيكى و محيطى ئنى

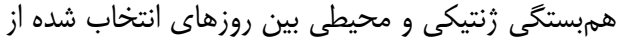

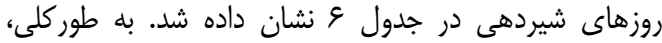

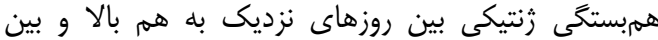

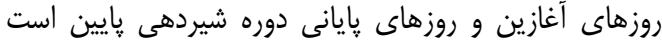

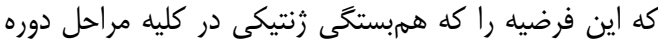

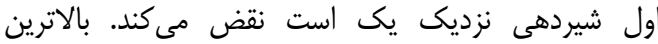

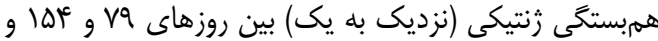

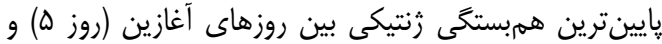

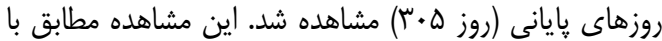

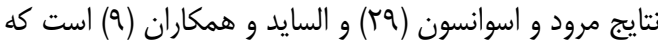

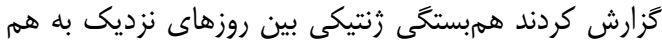

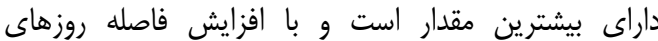

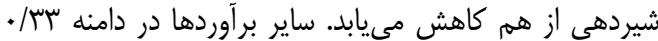

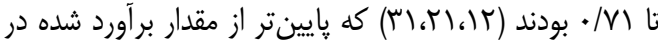

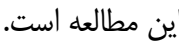

ميانخين وراثتيذيرى SCS دوره شيردهى اول كاوهاى

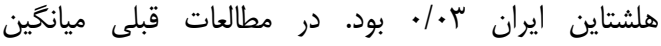

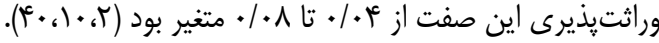

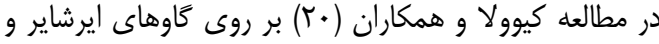

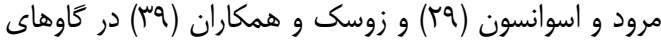

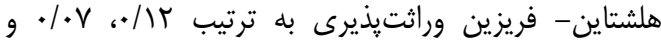

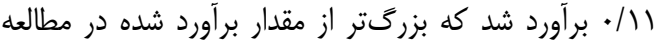

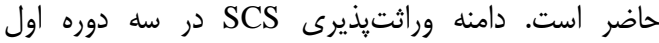

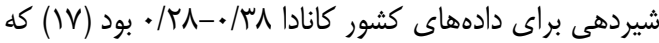

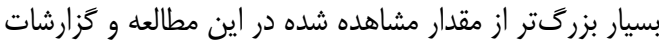

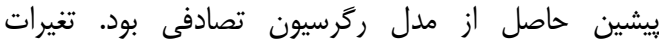

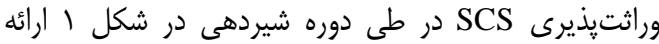

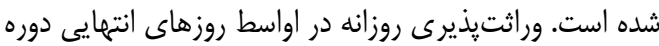

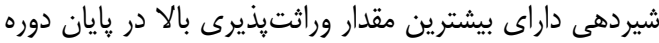

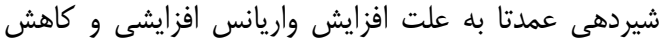

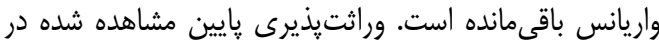

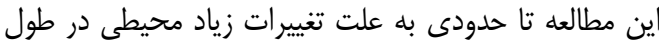

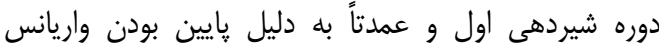

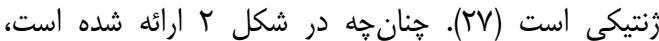

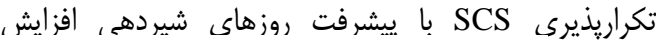

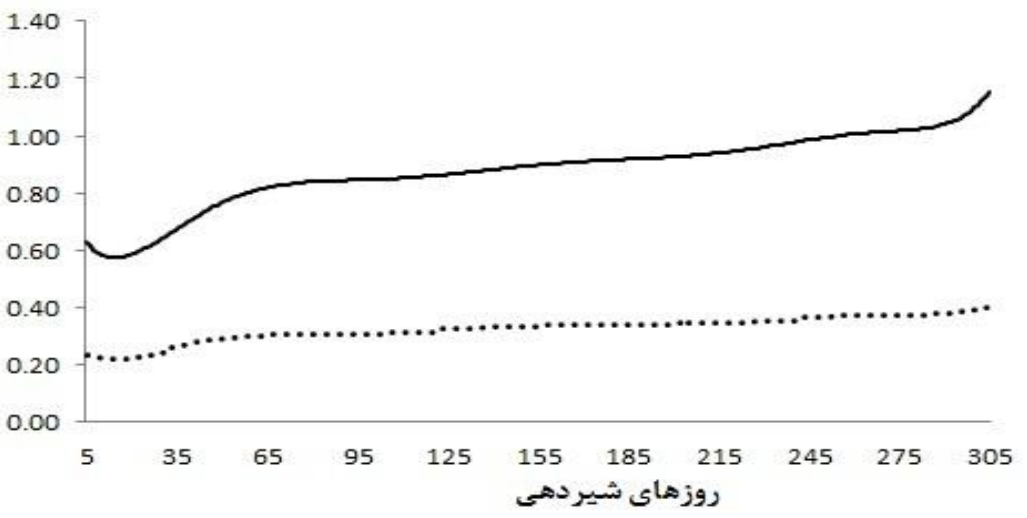

شكل r- واريانس محيطى دائمى (خط يِيوسته) و تكراريذيرى (نقطهجين) برآورد شده براى ركوردهاى روزآزمون SCS در طول دوره شيردهى Figure 2. Permanent environmental variance (continuous line) and estimated repeatability (dot) for test-day records of SCS over the lactation period

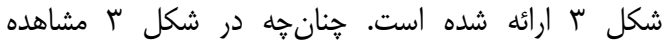

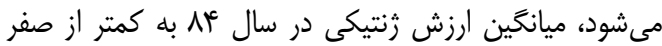

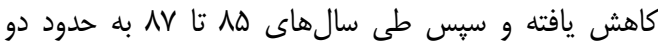

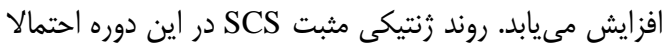

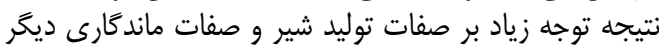

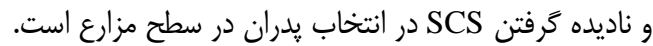

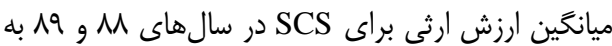

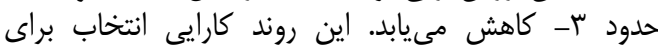

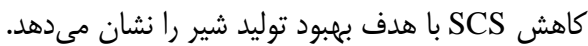

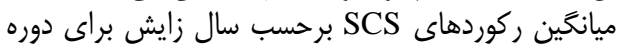

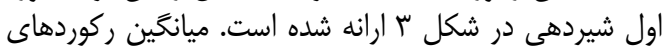

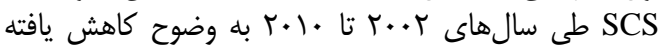

همجنين، همبستخى محيطى بين روزهاى نزديك به به هم

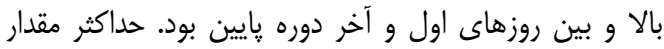

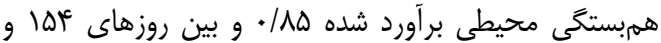

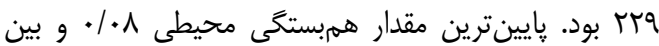

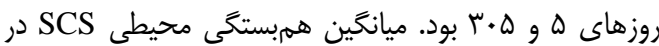

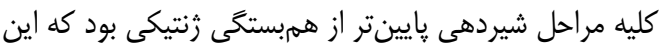

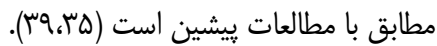
روند زنتيكى و فنوتيبي

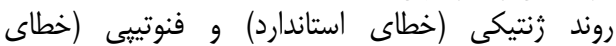

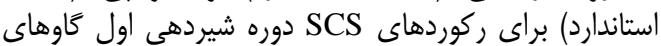

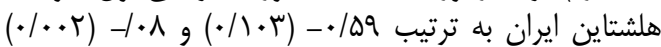

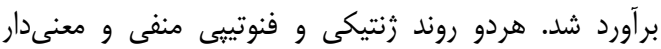

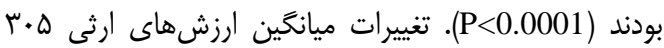

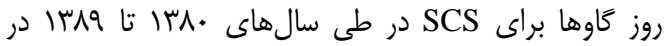




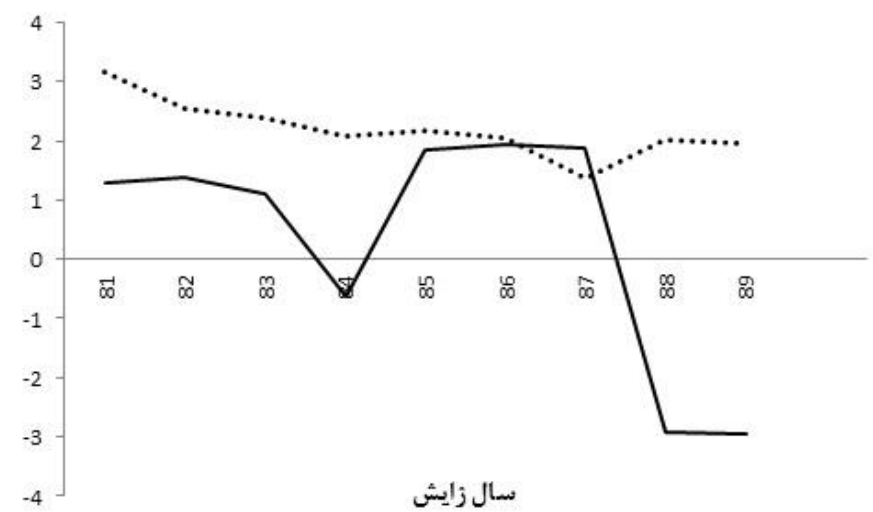

شكل س- ميانگين ارزش ارثى برآورد شده (خط ييوسته) و ميانگين فنوتيبى ركوردهاى روزآزمون (نقطه حين) SCS در طى سال هاى زايش Figure 3. Average estimated breeding value (continuous line) and phenotypic average of test-day records of SCS (dot) at calving years

وراثتيذيرى SCS در كاوهاى هلشتاين ايران يايين است.

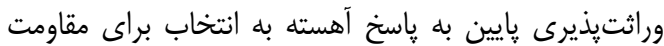

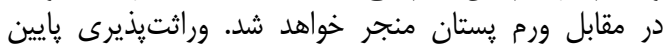

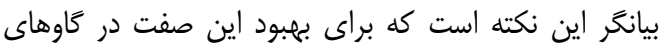

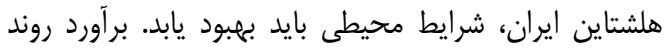

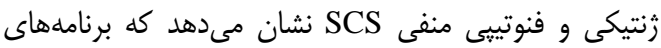

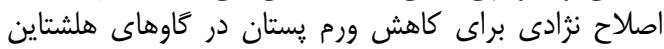
ايران در سالهاى اخير موفق بوده است.

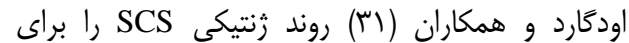

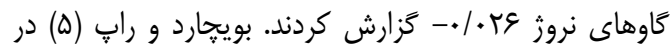

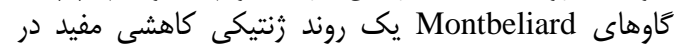

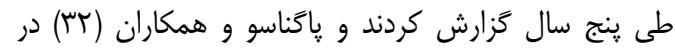

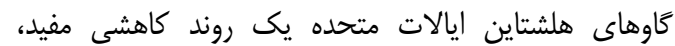
هرجنا كوجى

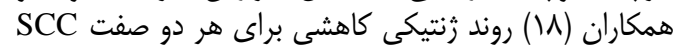

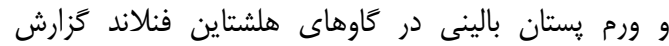
كردند. نتايج حاصل از تحقيت دان

جدول و- همبستخى هاى زنتيكى (بالاى قطر) و محيطى دائمى (يايين قطر) بين روزهاى انتخاب شده از دوره شيردهى Table 6. Genetic correlations (above diagonal) and permanent environmental correlations (below diagonal) among

\begin{tabular}{|c|c|c|c|c|c|}
\hline$r \cdot \omega$ & rTq & $1 \Delta F$ & vq & $\Delta$ & روز شيرهى \\
\hline$\cdot / \Delta V$ & $\cdot|\mathrm{A}|$ &.$/ 8$. &.$/ 09$ & 1 & Q \\
\hline$\cdot / M$ &.$/ 94$ & 1 & 1 & שא/. & vq \\
\hline$\cdot / M$ &.$/ 90$ & 1 &.$/ v 9$ &.$/ \mu$. & IQf \\
\hline.$/ 98$ & 1 & - / $\Delta \Delta$ &.$/ 8$. & G & rTq \\
\hline 1 & $\cdot / N$ & $\cdot / \Delta \Lambda$ &.$/ \$ \Delta$ & $.1 \cdot 1$ & $r \cdot \Delta$ \\
\hline
\end{tabular}

منابع

1. Akaike, H. 1973. Information theory and an extension of the maximum likelihood principle. Proceeding of $2^{\text {nd }}$ international symposium of information theory, Budapest, Hungary, 267-281.

2. Bakhtiarizadeh, M.R., M. Moradi Shahr Babak and A. Pakdel. 2010. Estimation of udder composite in the holstein population of Iran. Journal of Dairy Science, 93 (E-Suppl. 1): 597.

3. Bignardi, A.B., L. EL Faro, V.L. Cardoso, P.F. Machado and L.G. de Albuquerque. 2009. Random regression models to estimate test-day milk yield genetic parameters holstein cows in southeastern Brazil. Livestock Science, 123: 1-7.

4. Bloemhof, S., G. de Jong and Y. de Haas. 2009. Genetic parameters for clinical mastitis in the first three lactations of dutch holstein cattle. Veterinary Microbiology, 134: 165-171.

5. Boichard, D. and R. Rupp. 1997. Genetic analysis and genetic evaluation for somatic cell score in french dairy cattle. proceedings of the international workshop on genetic improvement of functional traits in cattle, uppsala, Sweden, June, Interbull Bulletin no, 15: 54-60.

6. Brotherstone, S., I.M.S. White and K. Meyer. 2000. Genetic modeling of daily yields using orthogonal polynomials and parametric curves. Journal of Animal Science, 70: 407-415.

7. Burnham, K.P. and D.R. Anderson. 1998. Model selection and inference: a practical informationtheoretic approach, springer-verlag, New York, USA, 488 pp. 
8. Carlen, E., E. Strandberg and A. Roth. 2004. Genetic parameters for clinical mastitis, somatic cell score, and production in the first three lactations of Swedish Holstein Cows. Journal of Dairy Science, 87: 3062-3070.

9. Elsaid, R., A. Sabry, M.S. Lund and P. Madsen. 2011. Genetic analysis of somatic cell score in danish dairy cattle using random regression test-day model. Livestock Science, 140: 95-102.

10. Ghavi Hossein-Zadeh, N. and M. Ardalan. 2011. Estimation of genetic parameters for milk urea nitrogen and its relationship with milk constituents in Iranian Holsteins. Livestock Science, 135: 274-281.

11. Ghavi Hossein-Zadeh, N., A. Nejati-Javaremi, S.R. Miraei-Ashtiani and H. Kohram. 2008. An observational analysis of twin births, calf stillbirth, calf sex ratio, and abortion in Iranian Holsteins. Journal of Dairy Science, 91: 4198-4205.

12. Haile Mariam, M., M.E. Goddard and P.J. Bowman. 2001a. Estimates of genetic parameters for daily somatic cell count of australian dairy cattle. Journal of Dairy Science, 84: 1255-1264.

13. Haile-Mariam, M., P.J. Bowman and M.E. Goddard. 2001b. Genetic and environmental correlations between test-day somatic cell count and milk yield traits. Livestock Production Science, 73: 1-13.

14. Jaffrézic, F., I.M.S. White, R. Thompon and W.G. Hill. 2000. A Link function approach to model heterogeneity of residual variances over time in lactation curve analyses. Journal of Dairy Science, 83: 1089-1093.

15. Jamrozik, J. and L.R. Schaeffer. 1997. Estimates of genetic parameters for a test day model with random regressions for yield traits of first lactation holsteins. Journal of Dairy Science, 80: 762-770.

16. Jamrozik, J., L.R. Schaeffer and F. Grignola. 1998. Genetic parameters for production traits and somatic cell score of canadian holsteins with multiple trait random regression model, Proceedings of $6^{\text {th }}$ WCGALP, 303-306 pp.

17. Jamrozik, J., L.R. Schaeffer, Z. Liu and G. Jansen. 1997. Multiple traits random regression test day model for production traits. proceedings of 1997 interbull meeting, Vienna, Austria, 43-47.

18. Juga, J., E.A. Mäntysaari and J. Pösö. 1999. Economic response to total merit selection in finnish ayrshire breeding. proceedings of the international workshop on EU concerted action on genetic improvement of functional traits in cattle (gift); breeding goals and selection schemes, wageningen, the netherlands, Interbull Bulletin, 23: 79- 87.

19. Kadarmideen, H.N. and J.E. Pryce. 2001. Genetic and economic relationships between somatic cell count and clinical mastitis and their use in selection for mastitis resistance in dairy cattle. Journal of Animal Science, 73: 229-240.

20. Kheirabadi, K. and S. Alijani. 2014. Comparison of two singles- and multiple trait random regression models in estimation of genetic parameters of production traits in holstein dairy cattle. Research on Animal Production, 5: 179-189.

21. Koivula, M., E. Negussie and E.A. Mäntysaari. 2004. Genetic parameters for test-day somatic cell count at different lactation stages of finnish dairy cattle. Livestock Production Science, 90: 145-157.

22. Lopez-Romero, P. and M.J. Carabano. 2003. Comparing alternative random regression models to analyse first lactation daily milk yield data in holstein friesian cattle. Livestock Production Science, 82: 81-96.

23. Lund, T., F. Miglior, J.C.M. Dekkers and E.B. Burnside. 1994. Genetic relationships between clinical mastitis, somatic cell count, and udder conformation in Danish Holsteins. Livestock Production Science, 39: 243-255.

24. Meyer, K. 1998. Estimating covariance functions for longitudinal data using a random regression model. Genetics Selection Evolution, 30: 221-240.

25. Meyer, K. 2011. Wombat a program for mixed model analyses by restricted maximum likelihood. univesity of New England, http://didgeridoo.une.edu.au/km/WOMBAT/WWW/manual.html.

26. Miller, R.H., M.J. Paape and L.A. Fulton. 1991. Variation in milk somatic cells of heifers at first calving. Journal of Dairy Science, 74: 3782-3790.

27. Mohammadpanah, M., H. Farhangfar and M. Bashtani. 2016. Genetic analysis of raw and energycorrected test day milk traits in iranian first lactation Holstein Cows. Research on Animal Production, 7: 153-162.

28. Mrode, R.A. and G.J.T. Swanson. 1996. Genetic and statistical properties of somatic cell count and its suitability as an indirect means of reducing the incidence of mastitis in dairy cattle. Animal Breeding Abstracts, 64: 847-857.

29. Mrode, R.A. and G.J.T. Swanson. 2003. Estimation of genetic parameters for somatic cell count in the first three lactations using random regression. Livestock Production Science, 79: 239-247.

30. Mrode, R.A., G.J.T. Swanson and M.S. Winters. 1998. Genetic parameters and evaluation for somatic cell count and its relationship with production and type traits in some dairy breeds in the United Kingdom. Journal of Animal Science, 66: 569-576.

31. Ødegard, J., J. Jensen, G. Klemetsdal, P. Madsen and B. Heringstad. 2003. Genetic analysis of somatic cell score in norwegian cattle using random regression test-day models. Journal of Dairy Science, 86: 4103-4114. 


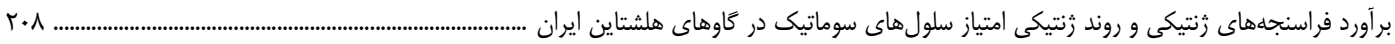

32. Pagnacco, G., F. Miglior, W.C. Zhang, J.C.M. Dekkers and E.B. Burnside.1994. Genetic evaluation for somatic cell count and relationship with inbreeding in Canadian Holsteins. Proceedings of the $5^{\text {th }}$ World Congress on Genetics Applied to Livestock Production, Guelph, Canada, 93-96 pp

33. Pösö, J. and E.A. Mäntysaari. 1996. Relationships between Clinical Mastitis, Somatic Cell Score and Production for the First Three Lactations of Finnish Ayrshire. Journal of Dairy Science, 79: 1284-1291.

34. Ptak, E., P. Brzozowski, W. Jagusiak and K. Zdziarski. 2007. Genetic parameters for somatic cell score for polish black-and-white cattle estimated with a random regression model. Journal of Animal and Feed Sciences, 16: 357-369.

35. Reents, R., J. Jamrozik, L.R. Schaeffer and J.C.M. Dekkers. 1995. Estimation of genetic parameters for test day records of somatic cell score. Journal of Dairy Science, 78: 2847-2857.

36. Reents, R., J.C.M. Dekkers and L.R. Schaeffer. 1994. Genetic Parameters of Test Day Somatic Cell Counts and Production Traits. Proceedings of The $5^{\text {th }}$ World Congress on Genetics Applied To Livestock Production, Guelph, Canada, 120-123.

37. Rodriguez-Zas, S.L., D. Gianola and G.E. Shook. 200. Evaluation of models for somatic cell score lactation patterns in Holsteins. Livestock Production Science, 67: 19-30.

38. Rupp, R. and D. Boichard. 1999. Genetic parameters for clinical mastitis, somatic cell score, production, udder type traits and milking ease in first lactation Holsteins. Journal of Dairy Science, 82: 2198-2204.

39. Rzewuska, K., J. Jamrozik, A. arnecki and T. Strabel. 2011. Genetic parameters of test-day somatic cell scores for the first three lactations of polish Holstein-Friesian Cattle. Czech Journal of Animal Science, 56: 381-389.

40. Sanjabi, M.R., A. Gholibaigi Fard, R. Vaez Torshizi, A. Lavaf and A.H. Ahadi. 2010. Genetic relationships between somatic cell counts, milk production and udder conformation traits in Iranian Holsteins. Journal of Dairy Science, 93 (E-Suppl. 1): 598.

41. SAS. 2002. SAS users Guide v.9.1: Statistics SAS institute inc., Cary, NC.

42. Wada, Y. and N. Kashiwagi. 1990. Selecting statistical models with information statistics. Journal of Dairy Science, 73: 3575-3582.

43. Yousefi-Golverdi, A., H. Hafezian, Y. Chashnidel and A. Farhadi. 2012. Genetic parameters and trends of production traits in Iranian Holstein population. African Journal of Biotechnology, 11: 2429-2435. 


\title{
Estimation of Genetic Parameters and Genetic Trends of Somatic Cell Score in Iranian Holsteins
}

\author{
Hassan Khanzadeh ${ }^{1}$ and Navid Ghavi Hossein-Zadeh ${ }^{2}$ \\ 1- Graduated M.Sc. Student, Department of Animal Science, University of Guilan \\ 2-Associate Professor, Department of Animal Science, (Corresponding author: nhosseinzadeh@guilan.ac.ir) \\ Received: November 11, 2014 \\ Accepted: May 30, 2015
}

\begin{abstract}
The objectives of present study were to estimate the genetic and permanent environmental covariance components and genetic and phenotypic trends of test-day records of somatic cell score (SCS) of the first lactation Iranian Holstein cows. Dataset included the 108995 test day records were collected by the Animal Breeding Center of Iran from 2001 to 2010. The GLM procedure of SAS software was used for fitting the fixed effects in the statistical models of analysis. All analyses were performed using the AIREML algorithm of WOMBAT software. 16 different random regression models were studied and compared based on Akaike's information criterion. Random regression model with Legendre polynomial functions of orders of 4, 4, 5 were chosen to fit fixed regression, additive genetic and permanent environmental effects, respectively, and residual variance was assumed in 4 heterogeneous levels during the lactation. Estimation of heritability was low (0.022 to 0.032$)$ that indicated this trait mostly affected by environmental factors. Genetic and environmental correlations between daily SCS were high for adjacent tests and low between the beginning and the end of lactation. The estimated environmental correlations were lower than the genetic correlations in all stages of lactation. Annual genetic and phenotypic trends were $-0.59 \pm 0.103$ and $-0.08 \pm 0.002$, respectively, for SCS trait. Both genetic and phenotypic trends for SCS were significant $(\mathrm{P}<0.0001)$. Negative genetic and phenotypic trends for SCS indicated that genetic programs for declining the incidence of mastitis in Iranian Holsteins were successful at recent years.
\end{abstract}

Keywords: Dairy Cow, Genetic Parameters, Genetic Progress, Mastitis, Random Regression Model 